\title{
Anti-Malarial Drug Development and Diffusion in an Era of Multidrug Resistance
}

\section{How Can an Integrated Health Framework Contribute to Sustainable Development Goal 3?}

Freek de Haan and Ellen H.M. Moors

\section{Introduction}

Sustainability has grown into a paradigm of grand societal and environmental challenges that cannot be ignored by governments, firms, and nongovernmental organizations (NGOs). At the same time, sustainability challenges these groups by requiring short-term investments while the benefits are for the long term. To reach a better environmental, economic, and social future for the world, the United Nations set targets for 2030 by introducing the sustainable development goals (SDGs). The close and mutual relation between sustainability and health is a central focus of SDG3, which aims to "ensure healthy lives and promote well-being for all at all ages" (UN 2015). The pharmaceutical industry is a major element of global healthcare and has the potential to play a key role toward the achievement of SDG3.

Sustainable healthcare, however, is challenged by flaws in the current pharmaceutical system. On the one hand, there is pressure from society to develop innovative medicines that address unmet medical needs. On the other hand, the development of these medicines is expensive while health budgets are inherently limited. Excessive health expenses will inevitably endanger access and affordability of treatment, and so there is also pressure to reduce costs and mitigate health expenses. As a result, pharmaceutical innovation systems are increasingly struggling to deliver new medicines for unmet medical needs at affordable prices (Moors et al. 2014).

Although these are challenges with a global reach, they are even more prominent for countries with limited access to resources, the so-called low and middle-income countries (LMICs). ${ }^{1}$ A substantial number of the SDG3 targets aim to improve

\footnotetext{
${ }^{1}$ Low-income economies are defined as those with a gross national income (GNI) per capita, calculated using the World Bank Atlas method, of $\$ 3,895$ or less; middle-income economies are those with a GNI per capita between $\$ 3,896$ and $\$ 12,055$; high-income economies are those with a GNI per capita of $\$ 12,056$ or more (IAMCR 2019).
} 
healthcare in LMICs. We highlight two of those targets. First, SDG target 3.3 proposes "to end the epidemics of AIDS, tuberculosis, malaria and neglected tropical diseases and to combat hepatitis, water-borne diseases and other communicable diseases by 2030." While the burden of these diseases are low in most affluent countries, they remain prevalent in LMICs (Bhutta et al. 2014). Second, SDG target 3.8 emphasizes the "achievement of universal health coverage, including financial risk protection, access to quality, essential health-care services and access to safe, effective, quality and affordable essential medicines for all” (UN 2015). Here again, people living in LMICs would benefit most from fulfillment of this target, as they are generally lagging behind when it comes to health coverage.

Unfortunately, there are important barriers to establishing a sustainable pharmaceutical system for LMICs. The development of new medicines is resource intensive but commercially unattractive when aiming for poor populations (Arrow et al. 2004; Ubben and Poll 2013). Another complicating factor is the number of stakeholder groups along the pharmaceutical value chain in LMICs. The interests of these stakeholders are often poorly aligned or even conflicting (Yadav et al. 2007). Finally, the healthcare systems in LMICs are heterogeneous in nature and often function suboptimally as they are fragmented and characterized by a shortage of resources (Mills 2014). As a result, medicines often fail to flow to the ones in need. Added together, these conditions complicate the achievement of global access to affordable medicine and the realization of SDG targets 3.3 and 3.8.

Although various solutions have been suggested in the literature to the challenges noted here, in practice a combination of reforms will be necessary to make the development and diffusion of medicines for LMICs sustainable (Moors et al. 2014). Such reforms include a larger, open-access role for public research institutes in the discovery, clinical testing, and safety evaluations of new drugs. Furthermore, innovative business models and collaborations are essential to address healthcare challenges that affect people in LMICs, and a broader view that encompasses policy solutions to tackle global health challenges is needed. This chapter applies an innovation systems perspective to analyze a worrying development that requires coordinated action at drug development, diffusion, and subsequent implementation stages. The challenge is that malaria parasites have started to develop multidrug resistance to the global first-line drug combinations (Imwong et al. 2017). As a result, malaria is becoming increasingly difficult to treat and innovative solutions toward a sustainable antimalarial drug development and diffusion system are urgently required.

Malaria is a tropical infectious disease caused by one-celled plasmodium parasites, which are transmitted through the bites of mosquitos. Of the four types of plasmodium parasites, p. falciparum is the most prevalent and by far the most deadly. In past decades, impressive progress was made in malaria control and the global burden has been reduced over $50 \%$ since the year 2000 . Still, nearly half a million people die each year because of the disease, mainly children under five in sub-Saharan Africa (WHO 2017). This is particularly tragic because malaria is well treatable and effective drugs do exist. Unfortunately, the most effective anti-malarial medicines often fail to 
reach the infected patient, especially in remote areas with limited access to quality healthcare.

Historically, the African continent has been the center of attention and funding for malaria control. However, this focus is shifting-at least partially-now that resistance to the global first-line anti-malarial drugs has emerged and is spreading through the Greater Mekong Subregion (GMS) in Southeast Asia. Although malaria incidence and mortality in the GMS is at historically low levels, these recent developments imply a serious threat to all gains against the disease (Phyo and von Seidlein 2017). Moreover, there is risk that resistance will also spread to South Asia and Africa, where the majority of malaria burden is situated. This could create a public health crisis because no alternative treatment with comparable efficacy against $p$. falciparum parasites is expected in the coming years (Wells et al. 2015). According to conservative estimations, the scenario of resistance spreading to Africa could cause over 100.000 excess deaths annually and the overall extra costs could be more than USD 400 million per year (Lubell et al. 2014).

Hence, new anti-malarial medicines and diffusion strategies are urgently required to restore anti-malarial efficacy in the GMS and to prevent the spread of resistance to other continents. These goals are particularly critical for achieving the SDG3 targets of ending the epidemics of malaria and achieving universal health coverage. In the remainder of the chapter, we focus on anti-malarial drug development and diffusion in the context of emerging drug resistance. By taking a systems perspective, we answer the research question: Which reforms in the anti-malarial drug development and diffusion system are required to address the emergence of multidrug resistance?

The next section presents a sustainable drug development and diffusion framework for analysis of innovative medicines in LMICs. Then we examine malaria in relation to the SDGs, explain the state of the art of malaria treatment, and provide the methodology for this chapter. The following section applies the integrated framework to evaluate the established anti-malarial drug development and diffusion system and discusses implications of emerging multidrug resistance. Finally, the findings are discussed and further policy implications are provided.

\section{Sustainable Drug Development and Diffusion Framework for LMICs}

While healthcare in most LMICs is still insufficient, the sustainability of healthcare systems in affluent parts of the world is also under pressure, owing to increasing health costs and an aging population. Although most inventions of new treatment modalities are made by academic institutions, drug development has almost exclusively become the activity of large pharmaceutical companies (Moors et al. 2014). The output of new drugs and vaccines, however, has been decreasing dramatically for the last decades. At the same time the prices of new drugs and vaccines have risen steadily, leading to access problems for many patients, especially in LMICs. 
In addition, the development and use of medicines is extensively institutionalized through regulations, guidelines, and standards. For example, randomized controlled trials to assess the safety and efficacy of medicines are costly but often mandatory for regulatory approval and market access (Howick 2011; Rafols et al. 2014; Timmermans and Berg 2003). Moreover, active ingredients can be expensive, investments are necessary to produce in line with international standards, and pharmaceutical companies require profit margins to invest in future R\&D and to remain commercially viable. The high development and production costs for innovative medicines provoke high end-user prices, which has been a prominent topic in societal debates. In resource restricted settings, the prices of innovative medicines are often too high for governments and patients. As a result, governments and patients in LMICs remain dependent on donor subsidies or lack the access to essential medicines. Moreover, counterfeits (fake medicines) have been entering the medicine market in many settings (Dondorp et al. 2004; Newton et al. 2001). Furthermore, cultural habits of citizen communities, perceived side effects, and inadequate information provision negatively influence the willingness of patients to take drugs (O'Connell et al. 2012). This is all leading to availability, affordability, accessibility, and acceptability problems for many patients in LMICs. Table 13.1 provides an overview of the central concepts for a sustainable pharmaceutical system in LMICs.

Accordingly, system reforms are necessary to sustainably address medical needs of people living in LMICs. Disruptive reform of the current pharmaceutical innovation system would offer alternative ways of developing drugs. While there is still a strong focus on fixing the flaws in the current system, in practice, a combination of reforms is necessary to make drug development and distribution to patients in LMICs sustainable. Sustainable drug development is described as a process in which medicines are accepted, safe, affordable, and accessible by the ones in need (Nwaka and Ridley 2003). Additionally, sustainability requires responsible deployment of scarce resources to reduce negative future effects such as accelerated development of drug resistance. The patent system, short-term incentives and reward systems, and the lack of capacity of academic institutions to develop innovative drugs are associated with an unsustainable system (Moors et al. 2014; Munos 2009). At the patient levels unsustainability is provoked by availability of low-quality drugs and inadequate utilization of drugs.

Sustainable access to medicine requires a multisectoral approach involving governments, the pharmaceutical industry, NGOs, and multilateral organizations (Meijer et al. 2013). Drug development, then, asks for open transfer and new forms of cooperation through, for example, public private partnerships (PPPs). Innovative and market focused policy approaches are required to improve access to medicines in LMICs (Waning 2011). These should involve sustainable funding, efficient drug regulation and registration practices, reimbursement programs, and effective supply of medicines to both public and private outlets (Arrow et al. 2004; Yadav et al. 2007). 
Table 13.1 Overview concepts of sustainable drug development and diffusion

\begin{tabular}{|c|c|}
\hline Concept & Description \\
\hline Availability & $\begin{array}{l}\text { Initiating and performing R\&D } \\
\text { to address unmet medical needs. } \\
\text { Sustainable drug development } \\
\text { should aim to create open } \\
\text { access to share knowledge and } \\
\text { to stimulate open innovation } \\
\text { for drugs, for example through } \\
\text { public-private consortia. } \\
\text { Availability also involves } \\
\text { capacity issues within regulatory } \\
\text { health authorities and drug } \\
\text { registration processes to enable } \\
\text { market access. }\end{array}$ \\
\hline
\end{tabular}

Affordability

Accessibility
Innovative medicines should be affordable to governments and to patients in resource restricted settings. Pricing strategies (both in public and private sectors) should therefore take the economic possibilities of the patient population in consideration. External funding with subsidies and reimbursement can improve affordability of drugs. In order to become sustainable, this requires ongoing funding rather than incidental programs. Patents and product competition can also affect affordability.

Accessibility deals with the distribution of interventions throughout supply chains: from the central levels to health practitioners and patient populations. Usually access starts with inclusion of effective and safe medicines in both normative global guidelines and in national treatment guidelines. Sustainable access also requires functioning supply chains and sufficient health system capacity. Both public and private sector channels should be taken in consideration to become sustainable. Moreover, access to low-quality alternatives should be avoided on the medicine markets.
Anti-malarial S,T,I implications

Pragmatic short term approaches are required to tackle the spread of multidrug resistant malaria. Product development consortia can prelude the availability of more sustainable anti-malarial drugs. A promising example is the recent establishment of a consortium which aims to develop and test triple artemisinin combination therapies (TACT). Moreover, accelerated regulatory pathways may be required to ensure rapid availability of innovative medicines.

Subsidies such as the Global Fund ensure affordability of medicines in the public sectors of most malaria endemic countries. Eligibility to these subsidy schemes is also required for rapid and sustainable uptake of new anti-malarial medicines, such as TACT. However, the most significant challenges relate to private sectors affordability. In most settings, this requires significant system reformations.

Healthcare systems and drug supply chains in malaria endemic settings are heterogeneous in nature. Therefore, no single solutions toward sustainable access to anti-malarials exists. However, inclusion of more sustainable drugs, such as TACT, in global and national guidelines is a crucial first step. Moreover, investments in structural improvements of (private sector) drug distribution networks is required to ensure accessibility of sustainable anti-malarials. Active market regulation may be required to avoid access to outdated, substandard, or counterfeit alternatives. 
Table 13.1 Continued

\begin{tabular}{|c|c|c|}
\hline Concept & Description & Anti-malarial S,T,I implications \\
\hline Acceptability & $\begin{array}{l}\text { Drugs need to be accepted by } \\
\text { prescribers and patients. This } \\
\text { can be achieved by offering } \\
\text { drugs without too many side } \\
\text { effects and risks. Moreover, } \\
\text { the drugs should be adapted } \\
\text { to end-user preferences. This } \\
\text { requires user involvement and } \\
\text { strategic consideration about } \\
\text { demand issues such as branding } \\
\text { and packaging in an early } \\
\text { stage. Acceptability also deals } \\
\text { with information provision } \\
\text { and promotional activities to } \\
\text { inform prescribers and patient } \\
\text { populations. }\end{array}$ & $\begin{array}{l}\text { During development of new } \\
\text { anti-malarials, including TACT } \\
\text { end-users preferences such as } \\
\text { taste, tablet size, and perceived } \\
\text { side effects should be considered } \\
\text { at an early stage to facilitate } \\
\text { rapid uptake. Also appropriate } \\
\text { branding and packaging of the } \\
\text { new medicines is required. After } \\
\text { market introduction, training } \\
\text { and promotional activities can } \\
\text { be useful tools in informing } \\
\text { target populations about the risks } \\
\text { of malaria resistance and the } \\
\text { importance of switching to new } \\
\text { anti-malarials. }\end{array}$ \\
\hline
\end{tabular}

Source: Based on Moors et al. (2014); Munos (2009); Warren (2018).

\section{Malaria: A Multifaceted Global Health Issue}

Malaria is a mosquito-borne tropical disease caused by plasmodium parasites. Of the four types of plasmodium parasites, plasmodium falciparum is by far the most common and the most deadly type (WHO 2017). In the remainder of the chapter, plasmodium falciparum malaria will simply be referred to as malaria.

\subsection{Malaria and the SDGs}

Major improvements have been made in global malaria control since the year 2000 . The burden has been reduced by more than half, and malaria has been eliminated in 19 countries. Moreover, global investments in malaria management and control have more than doubled between 2005 and 2014 (Cibulskis et al. 2016).

Nonetheless, malaria is still a significant health issue with 3.4 billion people in 91 countries at risk of the disease. Each year, more than 200 million people are infected with malaria and the disease takes an estimated 445,000 deaths per year (WHO 2017). This is unacceptably high when considering that malaria is well preventable and treatable. Malaria mortality is mainly prevalent in children under five years of age in sub-Saharan Africa, the region with highest malaria transmission. These young African children are most vulnerable to malaria because they have not yet acquired any form of immunity toward the disease, in contrast with adolescents and adults. In settings with lower transmission rates, including the GMS, malaria is less of a 
pediatric disease but also affects adults, often forest workers and migrant populations in border areas (WHO 2016).

The clinical representation of a malaria infection is usually classified into two levels of intensity: uncomplicated malaria and severe malaria. Uncomplicated malaria refers to a common malaria infection where the patient presents febrile symptoms such as headache, vomiting, chills, and diarrhea. These symptoms overlap with many other tropical diseases (Luxemburger et al. 1998). Hence, malaria is often under- or overdiagnosed when reliable diagnostics are not available. Symptoms of severe malaria include coma, organ failure, and eventually death (Bartoloni and Zammarchi 2012). A case of severe malaria requires intravenous medication for direct relief of symptoms, while global guidelines recommend a three day oral treatment for uncomplicated malaria (WHO 2017).

The ongoing malaria challenges have explicitly been addressed in the SDGs. The SDG target 3.3 aims to end the epidemics of malaria and other (infectious) diseases by 2030. However, malaria is a poverty-related disease and the disease also affects other areas of sustainable development. Reducing the malaria burden could also benefit other sustainability goals including poverty (SDG1) and inequality reduction (SDG10) and improving education through reducing disease related absence (SDG4). An increasing malaria burden because of multidrug resistance would simultaneously affect these goals in a negative manner. Aligned with the SDGs, WHO formulated a global technical strategy (GTS) 2016-2030 (see Table 13.2). This GTS report suggests ways to reduce global malaria mortality and case incidence by $90 \%$ in 2030, with intermediate objectives for the years 2020 and 2025 (WHO 2016). Moreover, malaria should be eliminated in at least 35 countries and, importantly, re-establishment of the disease needs to be prevented. These milestones seem ambitious and we are not yet on track to achieving them (WHO 2018).

A number of strategies and interventions are essential in malaria control and in proceeding toward the SDG and GTS targets. First, preventive measures such as insecticide treated bed nets (ITN) and indoor residual spraying (IRS) have proven extremely effective in improving malaria control (Bhatt et al. 2016). Second, in response to the overlap in symptoms between malaria and other diseases, WHO now recommends diagnostic confirmation of all suspected cases of malaria before administering treatment. Malaria diagnosis is typically conducted by means of microscopy or rapid diagnostic tests (RDT). Global availability of effective medicines is another strategic cornerstone for effective malaria control. These days, the treatment of uncomplicated falciparum malaria globally relies on a three-day oral course of artemisinin combination therapies (WHO 2001).

Malaria surveillance is another fundamental cornerstone for management of the disease. Surveillance refers to the generation of data in order to identify outbreaks and populations at risk, but also to target interventions and to evaluate control programs. However, such data are often considered limited or unreliable owing to poor information technology structures, lack of administrative traditions in LMICs, and 
Table 13.2 Global technical strategy for malaria 2016-2030

\begin{tabular}{lllll}
\hline & \multicolumn{2}{c}{ Milestones } & & Targets \\
\cline { 2 - 3 } \multicolumn{1}{c}{ Goals } & 2020 & 2025 & & 2030 \\
\hline $\begin{array}{l}\text { Reduce malaria mortality rates globally } \\
\text { compared with 2015 }\end{array}$ & $\geq 40 \%$ & $\geq 75 \%$ & $\geq 90 \%$ \\
$\begin{array}{l}\text { Reduce malaria case incidence globally } \\
\text { compared with 2015 }\end{array}$ & $\geq 40 \%$ & $\geq 75 \%$ & & $\geq 90 \%$ \\
$\begin{array}{l}\text { Eliminate malaria from countries in } \\
\text { which malaria was transmitted in 2015 }\end{array}$ & $\geq 10$ countries & $\geq 20$ countries & $\geq 35$ countries \\
$\begin{array}{l}\text { Prevent re-establishment of malaria in all } \\
\text { countries that are malaria-free }\end{array}$ & $\begin{array}{l}\text { Re- } \\
\text { establishment } \\
\text { prevented }\end{array}$ & $\begin{array}{l}\text { Re- } \\
\text { establishment } \\
\text { prevented }\end{array}$ & $\begin{array}{l}\text { Re- } \\
\text { establishment } \\
\text { prevented }\end{array}$ \\
\hline
\end{tabular}

Source: WHO (2016).

the absence of central coordination. Therefore, malaria surveillance is put on the agenda as one of the cornerstones for achieving the GTS goals (WHO 2016).

\subsection{Malaria Treatment: The State of the Art}

Artemisinin combination therapy (ACT) constitutes the regime of first-line treatment for uncomplicated malaria throughout the world. ACT combines an artemisinin derivative, the most potent class of anti-malarial drug, and a less aggressive but longer acting partner drug. The rationale behind deployment of combination therapies is to synergize efficacy of both individual compounds while, at the same time, risk of parasites developing resistance to the individual compounds is being reduced (White 1999).

ACT has only been available since the beginning of this millennium. For decades, the anti-malarial market had been dominated by chloroquine, a cheap and widely available drug costing approximately USD \$0.10 per dose (Arrow et al. 2004). Unfortunately, chloroquine resistance emerged in Asia and spread throughout the world. In response, malaria endemic countries switched to alternative first-line treatments such as sulfadoxine-pyrimethamine and mefloquine, but soon these replacement drugs started to fail as well (Faurant 2011). In the late 1990s there was only one anti-malarial compound left without declining efficacy: artemisinin, an extract of the Chinese sweet wormwood plant. It was clear that the future of malaria treatment had to rely on this ingredient and its derivatives (artesunate, artemether, dihydro-artemisinin).

The artemisinin derivatives are extremely potent in eliminating malaria parasites and in rapidly reducing the symptoms of a malaria infection. However, the compounds also have a short plasma half-life, which means that it is rapidly removed from 
the body. As a result, chances of parasites surviving the treatment are high, which may subsequently lead to recrudescence of the infection. Moreover-and this is considered an even larger threat-the short plasma half-life significantly reinforces the risk of drug resistance emerging (White 1999). These resistance mechanisms are a consequence of genetics and natural selection when the treatment regime fails to eliminate all parasites, for example because of inappropriate drug use (Phyo et al. 2016).

Given the fact that artemisinin is the last available anti-malarial and is crucially important in the battle against malaria, there has been a general consensus that artemisinin resistance would have to be avoided at all costs. This led to the guiding principle that artemisinin and its derivatives ought to be exclusively deployed in combination with a partner drug (usually an "older" anti-malarial with a history of resistance) (WHO 2001). The rationale is that the artemisinin compound of the ACT would reduce the majority of the parasite burden within the first three days of treatment. The partner drug component eliminates the remaining parasites in the following days and prevents recrudescence of the infection. WHO recommends a three-day oral course of ACT in all areas of resistance to traditional anti-malarials since the year 2001. In 2006, this was further extended to all cases of diagnostically confirmed uncomplicated malaria (WHO 2006).

Upon this policy change and when the transition to ACT was started, the global anti-malarial market was rapidly polluted by dangerous compounds that did not meet quality requirements. Artemisinin was still often being prescribed without partner drug, despite the risk of resistance, but also substandard and even counterfeit ACTs became commonly available on the market (Bosman and Mendis 2007; Newton et al. 2001). In response, WHO stressed that the prescription of artemisinin monotherapies (without a partner drug) should be avoided to reduce risk of artemisinin resistance. Also, unfinished courses of ACT and the use of drugs that did not meet quality standards were strongly discouraged in global treatment guidelines (WHO 2006). Moreover, the development of quality assured, fixed-dose ACT (the individual components combined in one pill) was initiated. The idea was that fixed-dose ACT would improve adherence to the full treatment regime and avoid the selective intake of compounds, as was common with coblistered drug combinations.

Despite all these preventive measurements, artemisinin resistance has emerged in Cambodia, and is spreading throughout the GMS (Imwong et al. 2017; Noedl et al. 2008; Phyo et al. 2016). Along the way, artemisinin resistant parasite lineages also managed to acquire resistance to partner drugs. As a result, malaria has become difficult to treat in the region. Switching to other ACT combinations has proven to be only a short-term solution before resistance to the new combination is acquired (Dondorp et al. 2017). Unfortunately, new anti-malarial compounds are still years away in the drug pipeline (Wells et al. 2015). Therefore, pragmatic, short term solutions are rapidly required to tackle the direct, urgent threat.

Potential short term solutions are to extend ACT intake from three to seven days, or to deploy multiple first-line ACTs in a region at the same time (Boni et al. 2016; Dondorp et al. 2017; Phyo and von Seidlein 2017). However, malaria experts consider 
a transition to triple combinations of currently prescribed compounds to be the most feasible short- to middle-term solution. Deployment of these triple artemisinin combination therapies (TACT) could provide direct relieve in the GMS, where double combinations are increasingly failing. Moreover, TACT could prevent spread and the de novo emergence of artemisinin and partner drug resistance in South Asia and Africa. In the long term, new active compounds will be required in the battle against malaria and to achieve the SDG3 and WHO's GTS goals.

\section{Methods}

This chapter takes a systems perspective and applies the sustainable framework for drug development and diffusion to the problem of emerging anti-malarial drug resistance. The qualitative study is based on desk research, including peer reviewed and grey literature review, and on expert consultations. Peer-reviewed literature was accessed via the Web of Science search engine. A broad search terminology was used to understand the anti-malarial drug development and diffusion systems and the implications of drug resistance. An example of such a search query is ("development" or "implementation" or "diffusion" or "transition") and ("artemisinin" or "artemether" or "artesunate" or "dihydroartemisinin") or ("ACT" and "malaria"). For each publication, the titles and abstracts were examined. The full content of papers that were subjected to relevant topics for anti-malarial drug development and diffusion was scrutinized. Papers that did not meet these criteria (e.g., in-vitro/vivo clinical studies, gene detection studies) were excluded. Moreover, references of all papers were checked for additional publications of interest. Typical journals of relevant studies included Malaria Journal, the New England Journal of Medicine, Drug Discovery Today, and Nature. Grey literature reports and websites were also identified through reference checks and by using similar search terminology in the search engine Google. Examples of selected grey literature are the yearly WHO malaria reports and the websites of the Worldwide Antimalarial Resistance Network (WWARN) and of the Medicines for Malaria Venture (MMV).

Additional insights were generated through expert consultations. Those consultations were conducted with individuals who have been involved professionally in the development, diffusion, and/or supply of (anti-malarial) medicines in LMICs. The experts were consulted in the period August-November 2018 and included representatives from academia and research based consultancy who are or have been active in projects related to anti-malarial drug development and diffusion. They were asked to share their experiences and opinions regarding the availability, affordability, accessibility, and acceptability of anti-malarial medicines. Where relevant to the background of the expert, specific consultation about these themes in light of drug resistance was requested.

The selected literature was screened for relevant topics regarding the established anti-malarial drug development and diffusion systems or to implications of emerging 
multidrug resistance to this system. Topics were then categorized according to the components of the sustainable drug development and diffusion framework. Moreover, when a topic was relevant to more than one category, this connection was further explored and explicated. The same process of data analysis was also carried out for data generated through the expert consultations. Finally, the results of the desk research were structured and will be presented in the next section. Each subsection first provides a general overview and relates the established subsystems for ACT to one of the framework components. The analysis then evaluates the situation of emerging of multidrug resistance. Finally, the implications of these developments on sustainable drug development are discussed. Interdependence between the framework components is specified throughout each subsection.

The GMS is a region in Southeast Asia and consists of six countries: Thailand, Cambodia, Myanmar, Vietnam, Laos, and China (Yunnan province). The malaria burden in the region has been strongly reduced in the last years: the number of malaria deaths declined 84\% between 2012 and 2015 (WHO 2015). These gains are mainly attributed to improved access to effective interventions for malaria prevention, diagnosis, and treatment. Despite these recent improvements, the GMS is also the region where resistance to artemisinin and partner drugs has developed and is spreading. Innovative responses are required to restore anti-malarial efficacy in the region. At the same time, the spread of resistance to South Asia and Africa, where the majority of the malaria burden is situated, could have a large public health impact. This situation touches upon many challenges for sustainable drug development for LMICs.

\section{Toward a Sustainable System of Anti-Malarial Drug Development and Diffusion}

Earlier in this chapter, a framework for sustainable drug development and diffusion in LMICs was developed. The present section applies this framework to provide an evaluation of the anti-malarial drug system following the framework components availability, affordability, accessibility, and acceptability. For each component, a general overview of the established system for ACT, the current first-line anti-malarial drugs, is provided. Then the implications of emerging multidrug resistance are evaluated. Finally, lessons from the integrated framework are translated into policy implications toward a sustainable system of anti-malarial drug development and diffusion, helping to meet the achievement of the SDG3 targets.

\subsection{Availability}

Traditionally, research based pharmaceutical companies are the institutions that develop innovative medicines and bring them to the market. However, the development 
of new medicines is resource intensive while the prospective returns when developing drugs for poor populations is low. Hence, for a long period of time, pharmaceutical companies have failed to address many neglected and poverty related diseases. This is well reflected by the history of anti-malarial medicines (Ubben and Poll 2013). Nearly all medicines that have become first-line treatments in recent history have been the product of governmental research projects (MMV 2016). Remarkably often they have been developed by military institutions during wartime. Examples have been chloroquine and sulfadoxine-pyrimethamine during the Second World War, and mefloquine during the Vietnam War. All these "traditional" anti-malarial medicines appeared relatively cheap to produce and were soon adopted in portfolios of pharmaceutical companies, including Indian generic manufacturers.

The anti-malarial drug development system appeared unsustainable, however, when resistance had emerged to all these traditional drugs at the end of the last millennium and a rapid transition to artemisinin-based combination therapies was required. At the time, artemisinin was only cultivated on a small scale and ACTs were not yet commercially produced. Because of the lengthy cultivation and extraction process of artemisinin, the estimated production price of ACT was twenty- to fiftyfold that of chloroquine (Arrow et al. 2004). As a result, ACT was considered unaffordable for patients and governments in most malaria endemic settings.

Despite the urgent need, most research based pharmaceutical companies were initially reluctant to invest in ACT, although some coblistered combinations were introduced (Ubben and Poll 2013; Wells et al. 2013). The only exemption at the time was Novartis, a large Swiss pharmaceutical company, which had a fixed-dose combination of Artemether and Lumefantrine (AL) in their pipeline. This first fixed-dose ACT was introduced in 2001 under the brand name Coartem ${ }^{\circledR}$ (Spar 2008). In the following years Coartem ${ }^{\circledR}$ was added to the $\mathrm{WHO}$ list of essential medicines and received regulatory prequalification approval, which enabled its entry on the global anti-malarial market.

A few years earlier, two organizations were established to extend the anti-malarial pipeline with fixed-dose ACTs. The Medicines for Malaria Venture (MMV) initiative started as a collaboration between multiple European governments and philanthropic organizations. The mission of MMV was to reduce the burden of malaria by discovering, developing, and delivering new effective and affordable antimalarial drugs (MMV 2016; Ubben and Poll 2013). Moreover, Drugs for Neglected Diseases initiative (DNDi) was founded by the MSF Access Campaign. Similar to MMV, it established a collaborative, needs-driven, not-for-profit drug research and development (R\&D) organization. Its aim was to develop new or improved treatments for neglected diseases, including malaria (DNDi 2015). In 2015, DNDi transferred all their malaria related activities to MMV.

These nonprofit organizations aimed to developed new medicines for resource restricted populations by combining expertise and capabilities from industry, academia, and NGOs. Funding came from governmental and philanthropic resources, and these partnerships led to the introduction of several fixed-dosed ACTs between 
2008 and 2012 (Bompart et al. 2011; Spar 2008; Wells et al. 2013). Different regulatory pathways were pursued, and most of these newly introduced ACTs received WHO prequalification status. Moreover, patent rights were waived for all the fixed-dose ACTs that were launched through these partnerships. This enabled generic pharmaceutical companies to enter the market, eventually leading to price reductions (Orsi et al. 2018). For example, a fixed-dosed combination of artesunate and mefloquine (AS-MQ) has been produced by Indian Cipla since 2008 after a technology transfer by the Brazilian pharma company Farmanguinhos (Wells et al. 2013).

Now that ACT efficacy has started to decline because of resistance, new medicines are again urgently required. Product development partnerships such as those managed by DNDi and MMV have proven to be very useful in managing the development of need-based medicine. They have successfully introduced a number of effective ACTs and it is likely that the near future pipeline of anti-malarials will also have to rely on partnerships. Therefore, they should be considered as important instruments in addressing unmet medical needs in LMICs and achieving the SDG3 targets of ending the epidemics of malaria (target 3.3) and of ensuring universal health coverage by providing access to safe, effective, quality, and affordable malaria products (target 3.8). An encouraging development is therefore the recent establishment of a consortium to start the development of triple ACT or TACT. By combining compounds with reverse resistance mechanisms, these TACT combinations are considered the most feasible short-term solution to address artemisinin and partner drug resistance. Such innovative development models reduce development costs and thereby contribute to increased affordability of new drugs. Moreover, lessons should be learned from past experiences in acquiring regulatory approval and in strategically positioning new anti-malarials on the market. Standard regulatory procedures can, for example, be too lengthy to respond to the development of resistance and so accelerated regulatory pathways may be required. Considering distribution and market related issues in an early stage allows for strategically anticipating on challenges and may therefore improve acceptability and affordability of new medicines. Hence, we stress the importance of complementary, bottom-up studies throughout the development of need-driven medicines to improve prospects of rapid uptake.

\subsection{Affordability}

The development of medicines is inherently a lengthy and resource intensive process. To remain profitable, pharmaceutical companies put innovative medicines on the markets for prices that are often too high for poor populations. Reducing the costs of development through innovative business models can therefore be an important step toward cheaper medicines. Moreover, sustainable funding programs or procurement subsidies are required for innovative medicines to become affordable for populations in LMICs. 
In the early days of ACT, a first solution toward affordability came in the form of a memorandum between WHO and Novartis. Directly after the introduction of Coartem ${ }^{\star}$, an agreement was signed that Novartis would waive profit and deliver Coartem ${ }^{\otimes}$ at cost to WHO. WHO would then distribute the medicines to the governments of malaria endemic countries. In return, WHO would provide Novartis with a quarterly forecast of expected orders (Bosman and Mendis 2007; Spar 2008). This way, Novartis was guaranteed that they would not risk an unsold overproduction, and they could start scaling up production. Economies of scale further reduced the production costs when global demand increased and Novartis managed to exponentially scale up the production of Coartem ${ }^{\circledast}$ (Spar 2008).

The funding structure between Novartis and WHO became the standard for other ACTs that were introduced, including those launched by the product development partnerships. As a result, access to ACT within the governmental controlled public sectors significantly improved. However, ACT was still significantly more expensive than all previous generations of anti-malarials and external subsidies were required to ensure affordability (Arrow et al. 2004). Initially, the funding of public sector procurement was accounted for by multiple donors. These days, most public sector procurement procedures have been institutionalized by a few larger programs such as the Global Fund to fight Aids, Tuberculosis, and Malaria. The Global Fund guarantees sustainable funding to ensure ongoing access to affordable, quality-ensured medicines.

Although many patients do benefit from these subsidies, most patients in developing countries seek care in the private sectors. For many of them, ACT remained unaffordable even after the establishment of the Global Fund, and these problems are unfortunately still ongoing (Littrell et al. 2011). As a result, many patients who purchase their medicines in the private sector still receive outdated, low-, quality or even fake medicines.

Moreover, in many settings even the subsidized ACT remain unaffordable for patient populations. In LMICs, public insurance schemes are absent and healthcare is usually paid out of pocket by patients (Hopkins 2010). Moreover, medicine supply chains in LMICs are complex and consist of many different levels of procurement agents, national and regional wholesalers, traders, and dispensing outlets (Yadav 2009). Price markups throughout the supply chain are common, especially in the private sectors. Within such an environment, heavily subsidized medicines can still be unaffordable for populations who often have an income below the poverty line. This requires increased control over supply chains and touches upon effective accessibility and acceptability issues. In this way, these subsidized medicines are undermining the overall achievement of SDG3 regarding increased affordability of medicines for all in need.

Innovative anti-malarial medicines that are suitable to tackle resistance, such as TACTs, need to become available at affordable prices. Eligibility to subsidies to stimulate affordability of anti-malarial medicines, including the Global Fund, is closely related to the regulatory status of the product (availability) and should therefore be 
addressed in an early developmental stage. Moreover, and this remains a serious challenge, affordability at the level of end-users in the private sectors should be ensured. These systems challenges not only touch upon the anti-malarial drugs but also affect other diseases such as tuberculosis and HIV/Aids. Hence, achievement of SDG3 by ending (poverty-related) epidemics and realizing universal health challenge requires ongoing attention to affordability of essential medicines.

\subsection{Accessibility}

When analysing drug distribution in LMICs, a distinction is usually made between private versus public sector accessibility. Private sector supply chains in LMICs often consist of unregulated networks of intermediary traders before medicines reach the retailing outlets (Yadav 2009). As explained earlier, these intermediaries usually require a mark-up, and so the prices of ACTs are incremented throughout the supply chain. Private sector retailers include formal facilities such as pharmacies and private clinics, but also informal facilities such as general stores and drug traders that sell anti-malarials (Yadav et al. 2007). Within the government regulated public sectors, drugs rather tend to flow horizontally from national governments to the level of health districts and eventually to the retailing facilities. Public sector retailers tend to be more compliant to guidelines than their private counterparts (O'Connell et al. 2011). The public sector includes governmental hospitals and health clinics, but also community or village health worker programs (Ajayi et al. 2008). Important to note here is that health systems, drug supply chains, and medicine markets are heterogeneous in nature and strongly context- and country-dependent. Therefore, our analysis will mainly touch upon some general themes instead of going in-depth into a specific local situation.

Once fixed-dose ACTs were produced and registered, they had to be delivered to both public and private sector retailers and prescribers in malaria endemic settings. The important first step was that malaria endemic countries had to include ACT as first-line anti-malarial drug in their national treatment guidelines. This pace was catalyzed after WHO recommended ACT as global first-line treatment for uncomplicated malaria in 2001. Between 2001 and 2007, nearly all malaria endemic countries did so in response to WHO recommendations (Bosman and Mendis 2007). However, time delays from months to years were common between inclusion in treatment guidelines and the start of implementation programs to actively ensure access and promote the uptake of ACTs in local settings.

From the onset, countries experienced many challenges with the distribution of quality assured ACT to end-users. Weak coordination of private sector supply chains and dispensing outlets often prevented quality assured ACT from reaching the patient, while outdated, low-quality, or even counterfeit medicines remained present on the anti-malarial markets (O'Connell et al. 2011; Palafox et al. 2014). Unfortunately, these problems are still ongoing in many endemic settings, and this challenges the 
achievement of universal health coverage (SDG3.8) by preventing patients from receiving adequate treatment. Moreover, deployment of inadequate medicines, in particular artemisinin monotherapies, contribute to increased drug pressure which puts the ability to end the endemics of malaria (SDG3.3) at risk.

The access problems are especially worrying in the light of emerging artemisinin and partner drug resistance. Lack of accessibility of appropriate, sustainable drugs are, equally to a lack of affordability, likely to stimulate the use of low quality alternatives. This, in turn, increases the risk of resistance. Hence, investments in overall structures of drug distribution in LMIC, with a strong emphasis on private sectors, is required for a more sustainable system. To tackle anti-malarial drug resistance, an important first step is inclusion of sustainable, evidence-based and quality assured drugs in both global and national guidelines. This is directly related to the availability of such medicines and requires a viable drug pipeline in times of multidrug resistance. Beyond that, increased coordination and proper supply chain management, including procurement, stocking, and distribution, is essential to ensure access to sustainable medicines (Palafox et al. 2014). This also includes market regulation to prevent inadequate medicines from being available. Access to appropriate drugs is, logically, dependent on access to those drugs.

\subsection{Acceptability}

Finally, for drug development and diffusion to become sustainable, evidence-based medicines should be accepted and used rationally. However, many factors complicate acceptability and again these factors may be heterogeneous according to the setting. In general, issues such as perceived safety and side effects are known to affect the acceptability of medicines (Moors et al. 2014). However, in LMICs the willingness and ability to pay for treatment modalities are other important factors that affect acceptability of medicines (Mills 2014). As elaborated in previous subsections, this requires not only alternative business models for production but also well-functioning supply chains.

Many elements have been proposed to affect the acceptance of ACT at the population level including the level of education and knowledge, community perceptions, and cultural and socioeconomic factors (Littrell et al. 2011). However, there is little consensus on effective behavior change strategies to increase uptake of anti-malarial medicines (Smith et al. 2009). This may well relate to the diversity of settings, which all deal with locally different socioeconomic, cultural, and epidemiological situation, but also to local market conditions. A complicating factor toward a sustainable pharmaceutical system is that the interest of the individual patient in selecting appropriate medicines does not necessarily match the interest of the larger population (Arrow et al. 2004). A sick patient may want a cheap and rapid working drug (e.g., artemisinin monotherapies), while the interest of the larger population is that the patient uses the most sustainable drugs with the lowest risk of developing resistance. Such issues, 
which can even be reinforced by, for example, side effects of the sustainable medicine, are also subject to ethical debates.

Despite the diversity in the acceptability literature, there is some consensus that community engagement programs can be effective in increasing the acceptance of ACT, and therefore in achieving the SDG3 targets (Ajayi et al. 2008; Yeung et al. 2008). The same applies for promotion campaigns that include end-user perceptions, including branding and packaging (Novotny et al. 2016). For sustainable new medicines, such as TACT, these issues should be considered in an early stage to allow for strategic consideration and to anticipate on appropriate market positioning. Finally, information provision and educating providers and populations in malaria endemic settings on disease trends such as drug resistance can be useful to improve acceptability of new medicines. However, the availability of effective and sustainable drugs are a prerequisite to acceptability. Moreover, the accessibility and affordability compared to alternative treatment options are also likely to affect end-user perceptions and the acceptability of new drugs. The acceptance and appropriate use of sustainable medicines is essential in the battle against resistance and therefore deserves strategic consideration by conducting patient acceptance studies to match innovative products with end-user preferences.

\section{Discussion}

In this chapter, a framework for sustainable drug development and diffusion in LMICs was developed. The framework was then applied to evaluate a global health challenge: the emergence of resistance to the global first-line anti-malarial medicines. The chapter started with the question which system reforms are required to tackle resistance. What becomes clear is that the threat of resistance is multifaceted and that availability, affordability, accessibility, and acceptability of essential medicines is interrelated. We have shown that a system approach is required to tackle this global health challenge and to work toward a sustainable system of anti-malarial drug development and diffusion. This involves collective actions of stakeholder groups along the medicine value chain and should be aligned with the prevalent institutional landscapes (Yadav 2009; Moors et al. 2014).

The assessment elaborately addressed relevant topics for achieving sustainable healthcare (SDG3) and other interlinked SDGs. Particular focus areas were SDG target 3.3, which aims to end the epidemics of a number of diseases, including malaria, by 2030, and SDG target 3.8, which explicates goals for achieving universal health coverage. However, the pathway toward a sustainable anti-malarial development and diffusion system also affects other SDGs. Simultaneously, emerging multidrug resistance does not only threat the achievement of SDG3, but also of these other goals. We mentioned that a reduced malaria burden can be associated with higher productivity and therefore reduces poverty (SDG1), and at the same time reduces inequality between countries (SDG10). Moreover, in many settings malaria is a pediatric disease 
which mostly affects children. Being sick because of malaria will prevent them from going to school, and therefore reducing the malaria burden through STI approaches will improve education rates (SDG4).

To become sustainable and to tackle the threat of resistance, a number of adaptations in the pharmaceutical system are required. First, sustainable and innovative anti-malarials should be developed and rapidly made available to patients through regulatory pathways (Waning 2011). To realize this, the role of the pharmaceutical industry needs to be reconsidered. Poverty-related diseases are often considered commercially unattractive by pharmaceutical companies, although corporate responsibility can be an incentive. At the same time, these companies are extremely powerful in developing drugs because they are the ones with expertise and resources to do so (Access to Medicine Index 2018). To become sustainable and safeguard future R\&D efforts for resource restricted populations, alternative business models such as product development partnerships are required. By combining expertise and resources of both industry and academia, such partnerships can be established and prices of drug development can be reduced. Promising results have been achieved through innovative approach such as MMV and DNDi in managing the development of a number of fixed-dose anti-malarials (Bompart et al. 2011; Ubben and Poll 2013; Wells et al. 2013). In line with this, the recent establishment of the DeTACT project is an encouraging development in the battle against multidrug resistant malaria. This partnership, funded by the UK Department for International Development, aims to pragmatically combine current anti-malarial compounds into TACT and test them in multiple malaria endemic settings. The idea is that selecting compounds with reverse resistance mechanisms can extend the effective lifetime of current compounds. This way, TACT can benefit populations in malaria endemic regions until effective new medicines become available.

To achieve a sustainable system, affordability of next-generation anti-malarials needs to be ensured. Therefore, drugs should be designed and positioned to fit established market structures and preconditions of subsidy schemes. Establishment of subsidy programs such as the Global Fund have been an important step forward to public sector affordability. Particular attention should now be given to affordability in private sector outlets, which generally lag behind its public counterparts. This could involve increased control on private sector supply chains and legal action against disproportional markups and retail prices (Novotny et al. 2016).

Sustainable accessibility at patient levels starts with inclusion of appropriate antimalarials in global and national guidelines but is also related to efficient supply chains and market conditions (O'Connell et al. 2011). Defining a market positioning strategy to integrate sustainable medicines into established market structures and stimulate procurement of appropriate medicines should therefore be a central focus in the trajectory toward a sustainable system. This is even more important in an era of multidrug resistance, where utilization of inadequate drugs can increase drug pressure and lead to accelerated resistance. Finally, acceptability of appropriate drugs is essential in achieving a sustainable pharmaceutical system. This can be achieved by 
offering drugs without too many side effects and risks and by adapting drugs to enduser preferences. Promotional activities to inform populations about appropriate and rational drug use can also be effective tools (Novotny et al. 2016). We stress that bottom-up studies to investigate end-user perceptions should be an integrated element throughout the development and implementation of need-driven medicines.

Our analysis shows that the four framework components are interrelated. Affordability and accessibility of innovative medicines are affected by similar distribution and market dynamics. At the same time, ensuring availability in line with institutional requirements is required for affordability and accessibility. Finally, acceptability of medicines will only become an issue if availability, affordability, and accessibility are ensured. The interrelatedness of issues could also be reflected in the SDGs. Ending epidemics that are mostly prevalent in LMICs (SDG3.3) cannot be seen separately from universal access to healthcare (SDG3.8). The same applies to the malaria burden versus poverty (SDG1), education (SDG4), and equality between countries (SDG10). Moreover, water and sanitation conditions (SGD6) and global warming (SDG13) may affect the living conditions of mosquitos and therefore also affect malaria endemicity. Given the interrelations and multifaceted nature, we argue that systemic approaches should be at the root of tackling global health challenges.

\section{Conclusion}

An integrated health framework toward sustainable drug development and diffusion in LMICs was developed. The framework was applied to evaluate the situation of emerging anti-malarial drug resistance. Some policy implications follow from the analysis. First, business models such as product development partnerships can be powerful instrument and innovative approach in addressing medical needs that are neglected by industry. Therefore, targeted investments in such collaborations can be a useful tool in achieving the SDG3 targets. The second policy implication is that the effective transition to new medicines in LMICs involves many stakeholders and takes place in complex institutional landscapes. Considering demand, distribution, and market-related issues in an early stage of drug development allows for adequate positioning of new medicines to tackle global health challenges. Finally, availability, affordability, accessibility, and acceptability of innovative medicines are important concepts in improving global health and can contribute significantly to the implementation of the SDG3. They are interrelated and should be considered as integrated components of the drug development and diffusion system rather than as isolated themes.

\section{Acknowledgments}

The authors want to thank the participants of the interviews and the science and innovation management bachelor students in the course Sustainability, Health and 
Medical Technology of Utrecht University for their valuable input. This chapter was made possible by funding from the Department for International Development, UK.

\section{References}

Access to Medicine Index. (2018). https://www.accesstomedicinefoundation.org/access-tomedicine-index (accessed April 4, 2019)

Ajayi, I. O., Browne, E. N., Garshong, B., Bateganya, F., Yusuf, B., Agyei-Baffour, P., Doamekpor, L., Balyeku, A., Munguti, K., Cousens, S., and Pagnoni. F. (2008). Feasibility and acceptability of artemisinin-based combination therapy for the home management of malaria in four African sites. Malaria Journal 7, 1-9.

Arrow, K. J., Panosian, C. B., Gelband, H. (2004). Saving Lives, Buying Time: Economics of Malaria Drugs in an Age of Resistance. National Academies Press, Washington, DC.

Bartoloni, A., and Zammarchi, L. (2012). Clinical aspects of uncomplicated and severe malaria. Mediterranean Journal of Hematology and Infectious Diseases 4(1), e2012026.

Bhatt, S., Weiss, D. J., Cameron, E., Bisanzio, D., Mappin, B. and Dalrymple. K. (2016). The effect of malaria control on plasmodium falciparum in Africa between 2000 and 2015. Nature 526(7572), 207-211.

Bhutta, Z. A., Sommerfeld, J., Lassi, Z. S., Salam, R. A., and Das. J. K. (2014). Global burden, distribution, and interventions for infectious diseases of poverty. Infectious Diseases of Poverty 3(1), 1-7.

Bompart, F., Kiechel, J. R., Sebbag, R., and Pecoul, B. (2011). Innovative public-private partnerships to maximize the delivery of anti-malarial medicines: lessons learned from the ASAQ Winthrop experience. Malaria Journal 10(1), 143.

Boni, M. F., White, N. J. and Baird, J. K. (2016). The community as the patient in malariaendemic areas: preempting drug resistance with multiple first-line therapies. PLoS Medicine $13(3), 1-7$.

Bosman, A., and Mendis, K. N. (2007). A major transition in malaria treatment: the adoption and deployment of artemisinin-based combination therapies. American Journal of Tropical Medicine and Hygiene 77(6), 193-197.

Cibulskis, R. E., Alonso, P., Aponte, J., Aregawi, M., Barrette, A., Bergeron, L., Fergus, C. A., Knox, T., Lynch, M., Patouillard, E., Schwarte, S., Stewart, S., and Williams. R. (2016). Malaria: global progress 2000 - 2015 and future challenges. Infectious Diseases of Poverty 5(1), 1-8.

Drugs for Neglected Diseases Initiative (DNDI). (2015). The Successful Development of a Fixed Dose Combination of Artesunate Plus Amodiaquine Antimalarial. Drugs for Neglected Diseases Initiative, Geneva.

Dondorp, A. M., Newton P. N., Mayxay M., Van Damme W., Smithuis F. M., Yeung S., Petit A., Lynam A. J., Johnson A., Hien T. T., McGready R., Farrar J. J., Looareesuwan S., Day N. P. J., Green M. D., and White N. J. (2004). Fake antimalarials in Southeast Asia are a major impediment to malaria control: multinational cross-sectional survey on the prevalence of fake antimalarials. Tropical Medicine and International Health 9(12), 1241-1246.

Dondorp, A. M., Smithuis F. M., Woodrow, C., and von Seidlein, L. (2017). How to contain artemisinin- and multidrug-resistant falciparum malaria. Trends in Parasitology 33(5), 353-363.

Faurant, C. (2011). From bark to weed: the history of artemisinin. Parasite 18(3), 215-218.

Hopkins, S. (2010). Health expenditure comparisons: low, middle and high income countries. Open Health Services and Policy Journal 3(1), 111-117. 
Howick, J. H. (2011). The Philosophy of Evidence-Based Medicine. John Wiley and Sons, New York.

Imwong, M., Suwannasin, K., Kunasol, C., Sutawong, K., Mayxay, M., Rekol, H., Smithuis, F. M., Hlaing, T. M., Tun, K. M., van der Pluijm, R. W., Tripura, R., Miotto, O., Menard, D., Dhorda, M., Day, N. P. J., White, N. J. and Dondorp, A. M. (2017). The spread of artemisininresistant plasmodium falciparum in the Greater Mekong subregion: a molecular epidemiology observational study. The Lancet Infectious Diseases 17(5), 491-497.

International Association for Media and Communication Research (IAMCR) (2019). CountryIncome Classification for IAMCR Membership. https://iamcr.org/income (accessed April $8,2019)$.

Littrell, M., Gatakaa H., Evance I., Poyer S., Njogu J., Shewchuk S. T., Munroe E., Chapman S., Goodman C., Hanson K., Zinsou C., Akulayi L., Raharinjatovo J., Arogundade E., Buyungo P., Mpasela F., Adjibabi C., Agbango J., Ramarosandratana B., Coker B., Rubahika D., Hamainza B., Shewchuk, T., Chavasse, D., and O'Connell, K. A. (2011). Monitoring fever treatment behaviour and equitable access to effective medicines in the context of initiatives to improve act access: baseline results and implications for programming in six African countries. Malaria Journal 10, 327.

Lubell Y., Dondorp A. M., Guerin P. J., Drake T., Meek S., Ashley E., Day N. P. J., White N. J., and White L. J. (2014). Artemisinin resistance-modelling the potential human and economic costs. Malaria Journal 13, 452.

Luxemburger, C., Nosten, F., Kyle D. E, Kiricharoen, L., Chongsuphajaisiddhi, T., and White, N. J. (1998). Clinical features cannot predict a diagnosis of malaria or differentiate the infecting species in children living in an area of low transmission. Transactions of the Royal Society of Tropical Medicine and Hygiene 92(1), 45-49.

Medicines for Malaria Venture (MMV). (2016). Developing New Medicines for Case Management and Vulnerable Populations. MMV, Geneva.

Meijer, A., Boon, W. P. C., and Moors, E. H. M. (2013). Stakeholder engagement in pharmaceutical regulation: connecting technical expertise and lay knowledge in risk monitoring. Public Administration, 9(3), 696-711.

Mills, A. (2014). Health care systems in low- and middle-income countries. New England Journal of Medicine 370(6), 552-557.

Moors, E. H. M., Cohen, A. F., and Schellekens, H. (2014). Towards a sustainable system of drug development. Drug Discovery Today 19(11), 1711-1720.

Munos, B. (2009). Lessons from 60 years of pharmaceutical innovation. Nature Reviews in Drug Discovery 8, 959-968.

Newton P., Proux S., Green M., Smithuis F. M., Rozendaal J., Proux Prakongpan S., Chotivanich K., Mayxay M., Looareesuwan S., Farrar J., Nosten F., White, N. J. (2001). Fake artesunate in Southeast Asia. Lancet 357(9272), 1948-1950.

Noedl, H., Se, Y. Schaecher, K., Smith, B. L., Socheat, D., and Fukuda. M. M. (2008). Evidence of artemisinin-resistant malaria in Western Cambodia. New England Journal of Medicine 359(24), 2619-2620.

Novotny, J., Singh, A., Dysoley, L., Sovannaroth, S., and Rekol, H. (2016). Evidence of successful malaria case management policy implementation in Cambodia: Results from national ACTwatch outlet surveys. Malaria Journal 15, 194.

Nwaka, S., and Ridley, R. G., (2003). Virtual drug discovery and development for neglected diseases through public-private partnerships. Nature Reviews Drug Discovery 2(11), 919-928.

O’Connell, Gatakaa H., Poyer S., Njogu J., Evance I., Munroe E., Solomon T., Goodman C., Hanson K., Zinsou C., Akulayi L., Raharinjatovo J., Arogundade E., Buyungo P., Mpasela F., Adjibabi C. B., Agbango J. A., Ramarosandratansa B. F., Coker B., Rubahika D., Hamainza B., Chapman S., Shewchuk T., Chavasse D. (2011). Got ACTs? availability, price, market 
share and provider knowledge of anti-malarial medicines in public and private sector outlets in six malaria-endemic countries. Malaria Journal 10, 326.

O’Connell, Kathryn A., Samandari, G., Phok, S., Phou, M., Dysoley, L., Yeung, S., Allen, H., and Littrell. M. (2012). Souls of the ancestor that knock us out and other tales. a qualitative study to identify demand-side factors influencing malaria case management in Cambodia. Malaria Journal 11, 1-13.

Orsi, F., Singh, S., and Sagaon-Teyssier. L. (2018). The creation and evolution of the donor funded market for antimalarials and the growing role of southern firms. Science, Technology and Society 23(3), 349-370.

Palafox, B., Patouillard, E., Tougher, S., Goodman, C., Hanson, K., Kleinschmidt, I., Rueda, S. T., Kiefer, S., O’Connell, K.A., Zinsou, C., Phok, S., Akulayi, L., Arogundade, E., Buyungo, P., Mpasela, F., and Chavasse. D. (2014). Understanding private sector antimalarial distribution chains: a cross-sectional mixed methods study in six malaria-endemic countries. PLoS ONE 9(4), e93763.

Phyo, A. P., Ashley, E. A., Anderson, T. J. C., Bozdech, Z., Carrara, V. I., Sriprawat, K., Nair, S., McDew White, M., Dziekan, J., Ling, C., Proux, S., Konghahong, K., Jeeyapant, A., Woodrow, C.J., Imwong, M., McGready, R., Lwin, K. M., Day, N. P. J., White, N. J., and Nosten, F. (2016). Declining efficacy of artemisinin combination therapy against p. falciparum malaria on the Thai-Myanmar border (2003-2013): the role of parasite genetic factors. Clinical Infectious Diseases 63(6), 784-791.

Phyo, A. P., and von Seidlein, L. (2017). Challenges to replace ACT as first-line drug. Malaria Journal 16(1), 1-5.

Rafols, I., Hopkins, M., Hoekman, J., Siepel, J., O’Hare, A., Perianes-Rodrigez, A. and Nightingale, P. (2014). Big Pharma, little science? a bibliometric analysis on Big Pharma’s RandD decline. Technological Forecasting and Social Change 81, 22-31.

Smith, L. A., Jones, C., Meek, S., and Webster. J. (2009). Review: provider practice and user behavior interventions to improve prompt and effective treatment of malaria: do we know what works? American Journal of Tropical Medicine and Hygiene 80(3), 236-335.

Spar, D., and Delacey, B. J. (2008). The coartem challenge. Harvard Business School Case 706-037.

Timmermans, S., and Berg, M. (2003). The Gold Standard: The Challenge Of Evidence-Based Medicine. Temple University Press, Philadelphia.

Ubben, D., and Poll, E. M. (2013). MMV in partnership: ehe Eurartesim experience. Malaria Journal 12(1), 1-10.

United Nations. (2015) The Millennium Development Goal Report. https://sustainabledevelopment.un.org (accessed April 4, 2019).

Waning, B. (2011). Innovative approaches for pharmaceutical policy research in developing countries: the view through a market lens. PhD diss., Utrecht University.

Warren, M. (2018). Access to Medicine Foundation. Guest lecture, Utrecht University, bachelor course Sustainable Health and Medical Technology, September 27, 2018.

Wells, S., Graciela D., and Kiechel, J. R. (2013). The story of artesunate-mefloquine (ASMQ), innovative partnerships in drug development: case study. Malaria Journal 12(1), 1-10.

Wells, T. N. C., Hooft Van Huijsduijnen, R., and Van Voorhuis, W. C. (2015). Malaria medicines: a glass half full? Nature Reviews Drug Discovery 14(6), 424-442.

White, N. J. (1999). Antimalarial drug resistance and combination chemotherapy. Philosophical Transactions of the Royal Society B: Biological Sciences 354(1384), 739-749.

World Health Organization (WHO) (2001). Antimalarial Drug Combination Therapy. Report of a WHO Technical Consultation. World Health Organization, Geneva.

World Health Organization (WHO) (2006). WHO Briefing on Malaria Treatment Guidelines and Artemisinin Monotherapies. World Health Organization, Geneva. 
World Health Organization (WHO) (2015). Strategy for Malaria Elimination in the Greater Mekong Subregion (2015-2030). World Health Organization, Geneva.

World Health Organization (WHO) (2016). Global Technical Strategy for Malaria 2016-2030. World Health Organization, Geneva.

World Health Organization (WHO) (2017). World Malaria Report 2017. World Health Organization, Geneva.

World Health Organization (WHO) (2018). World Malaria Report 2018. World Health Organization, Geneva.

Yadav, P. (2009). Countering Drug Resistance in the Developing World: An Assessment of Incentives across the Value Chain and recommentations for Policy Interventions. Center for Global Development, Washington, DC.

Yadav, P., Sekhri, N., and Curtis. K. (2007). Barriers to access: an assessment of stakeholder risks and incentives in the value chain for artemisinin combination therapy (ACT) treatments. SSRN. doi.org/10.2139/ssrn.1008307

Yeung, S., van Damme, W., Socheat, D., White, N. J., and Mills. A. (2008). Access to artemisinin combination therapy for malaria in remote areas of Cambodia. Malaria Journal 7, 1-14. 